\title{
The progenitor of binary millisecond radio pulsar PSR J1713+0747 (Research Note)
}

\author{
W.-C. Chen ${ }^{1,2,3}$ and J. A. Panei ${ }^{4,5}$ \\ 1 Department of Physics, Shangqiu Normal University, 476000 Shangqiu, PR China \\ e-mail: chenwc@nju.edu.cn \\ 2 School of Physics and State Key Laboratory of Nuclear Physics and Technology, Peking University, 100871 Beijing, PR China \\ Key Laboratory of Modern Astronomy and Astrophysics (Nanjing University), Ministry of Education, 210093 Nanjing, PR China \\ 4 Facultad de Ciencias Astronómicas y Geofísicas, UNLP, Paseo del Bosque S/N, La Plata B1900FWA, Argentina \\ e-mail: panei@fcaglp.unlp.edu.ar \\ 5 Instituto de Astrofísica de La Plata (CCT La Plata), CONICET-UNLP, Argentina
}

Received 21 April 2010 / Accepted 10 January 2011

\section{ABSTRACT}

\begin{abstract}
Context. PSR J1713+0747 is a binary system comprising millisecond radio pulsar with a spin period of $4.57 \mathrm{~ms}$, and a low-mass white dwarf (WD) companion orbiting the pulsar with a period of 67.8 days. Using the general relativistic Shapiro delay, the masses of the WD and pulsar components were previously found to be $0.28 \pm 0.03 M_{\odot}$ and $1.3 \pm 0.2 M_{\odot}(68 \%$ confidence), respectively. Aims. Standard binary evolution theory suggests that PSR J1713+0747 evolved from a low-mass X-ray binary (LMXB). Here, we test this hypothesis.

Methods. We used a binary evolution code and a WD evolution code to calculate evolutionary sequences of LMXBs that could result in binary millisecond radio pulsars such as PSR J1713+0747.

Results. During the mass exchange, the mass transfer is nonconservative. Because of the thermal and viscous instabilities developing in the accretion disk, the neutron star accretes only a small part of the incoming material. We find that the progenitor of PSR J1713+0747 can be modelled as an LMXB including a donor star with mass 1.3-1.6 $M_{\odot}$ and an initial orbital period ranging from 2.40 to 4.15 days. If the cooling timescale of the WD is $8 \mathrm{Gyr}$, its present effective temperature is between 3870 and $4120 \mathrm{~K}$, slightly higher than the observed value. We estimate a surface gravity of $\log (g) \approx 7.38-7.40$.
\end{abstract}

Key words. stars: low-mass - pulsars: general - white dwarfs

\section{Introduction}

Since the first X-ray source Sco X-1 was discovered (Giacconi et al. 1962), it was proposed that the strong Galactic X-ray sources were accreting neutron stars or black holes in binary systems (Zeldovich \& Guseynov 1966; Novikov \& Zel'Dovitch 1966; Shklovskii 1967). These sources have significant higher flux in X-ray than other wavelengths. Over $90 \%$ of strong Galactic X-ray sources can be classified in two kinds as follows: high-mass $\mathrm{X}$-ray binaries and low-mass $\mathrm{X}$-ray binaries (LMXBs). The latter consists of a neutron star or a black hole and a low-mass donor star $\left(\lesssim 1-2 M_{\odot}\right)$.

The mechanisms that drive mass transfer in LMXBs depend on the initial separations of the binary components (Bhattacharya \& van den Heuvel 1991). In narrow systems with an orbital period $\left(P_{\text {orb }}\right)$ less than two days, mass transfer is driven by angular momentum loss due to gravitational radiation and magnetic braking. However, mass transfer is driven by the evolution of the secondary in relatively wide LMXBs $\left(P_{\text {orb }} \gtrsim 2 \mathrm{~d}\right)$. In LMXBs, mass accretion onto neutron star causes a magnetic field decay and spins it up to a short spin period $(\$ 20 \mathrm{~ms})$. It is believed that circular binaries including millisecond radio pulsar and $\mathrm{He}$ or $\mathrm{CO}$ white dwarfs (WDs) are formed as a result of long-term mass accretion (Alpar et al. 1982; Stairs 2004). The discovery of the first millisecond X-ray pulsar in LMXB SAX 1808.4-3658 strongly supports this scenario (Wijnards \& van der Klis 1998).
Through a survey for millisecond pulsars with the $305 \mathrm{~m}$ Arecibo radio telescope, Foster et al. (1993) have discovered PSR J1713+0747. This pulsar has a spin period of $4.57 \mathrm{~ms}$. It is in a circular orbit of 67.8 days and has a low-mass WD as companion. It has a weak magnetic field of $1.9 \times 10^{8} \mathrm{G}$ and a spin-down age of $8.9 \pm 1.0 \mathrm{Gyr}$, so is an older neutron star. By detecting the general relativistic Shapiro delay, the neutron star and WD were set up as the low limits for the masses of $1.2 M_{\odot}$ and $0.27 M_{\odot}$, respectively (Camilo et al. 1994).

Through observations for 12 years between April 1992 and May 2004, 343 pulse times of arrival for PSR J1713+0747 were recorded with uncertainties down 200 nanosecond. These timing data can result in the measurement of the Shapiro delay, which can constrain the mass of the WD $M_{2}$ and the inclination of the orbit $i$. The observational data constrained the masses of the pulsar to $1.3 \pm 0.2 M_{\odot}$ and the WD to $0.28 \pm 0.03 M_{\odot}(68 \%$ confidence, Splaver et al. 2005).

It is clear that PSR J1713+0747 is a recycled pulsar, because it gained mass and angular momentum from its donor star, the progenitor of the WD (Alpar et al. 1982). Assuming the mass of the WD is equal to the helium core mass of its progenitor, binary evolution theory has presented a peculiar relation between the orbital period $P_{\text {orb }}$ and the mass of WD $M_{2}$ (Rappaport et al. 1995; Tauris \& Savonije 1999; Podsiadlowski et al. 2002). According to this relation, Tauris \& Savonije (1999) obtained the range of the WD mass for PSR 
$\mathrm{J} 1713+0747,0.31 M_{\odot}<M_{2}<0.34 M_{\odot}$. Later on, $M_{2}$ was constrained in the range of $0.30-0.35 M_{\odot}$ (Pfahl et al. 2002). After adopting the predicted value by the theoretical relation of $P_{\text {orb }}-M_{2}$, the pulsar has a somewhat higher mass, $M_{1}=$ $1.53_{-0.06}^{+0.08} M_{\odot}(68 \%$ confidence) (Splaver et al. 2005).

Assuming that the neutron star accretes $10 \%$ of the material coming from the donor star, Benvenuto et al. (2006) have calculated the evolution of PSR J1713+0747 progenitor to fit its component masses and the orbital period. Their results show that the progenitor of PSR J1713+0747 may be an LMXB with initial masses of $M_{1}=1.4 M_{\odot}$ and $M_{2}=1.5 M_{\odot}$ and an initial orbital period of $P_{\mathrm{i}}=3.10 \mathrm{~d}(Z=0.02)$ or $P_{\mathrm{i}}=3.05 \mathrm{~d}(Z=0.01)$. Adopting a semi-analytic approach, Chen et al. (2006) have also performed binary evolution calculations to account for the component masses and the orbital period of PSR J1713+0747.

In this paper, where we employ a detailed stellar evolutionary code and a WD evolutionary code, we attempt to simulate the evolutionary history of the progenitor of binary millisecond radio pulsar PSR J1713+0747. The structure of this paper is organized as follows. In Sect. 2, we describe the stellar evolutionary codes, input physics in the binary evolution calculations, and the WD evolutionary code. Numerically calculated results for the evolutionary sequences of binary system resulting in the birth of PSR J1713+0747 are presented in Sect. 3. Finally, we discuss and summarize our results in Sect. 4.

\section{Stellar evolutionary code and input physics}

By employing an updated version of the stellar evolutionary code developed by Eggleton (1971), Eggleton (1972) (see Han et al. 1994; Pols et al. 1995), we investigated the evolution of LMXBs. The LMXBs consist of a main sequence donor star with a mass of $M_{2}$ and a neutron star with a mass of $M_{1}$. Radiative opacities are those from Rogers \& Iglesias (1992) and at low-temperature molecular opacities from Alexander \& Ferguson (1994). For the secondary star, we take a mixing-length parameter of $\alpha=2.0$ and an effective Roche lobe radius given by Eggleton (1983)

$$
\frac{R_{\mathrm{L}}}{a}=\frac{0.49 q^{2 / 3}}{0.6 q^{2 / 3}+\ln \left(1+q^{1 / 3}\right)},
$$

where $a$ and $q=M_{2} / M_{1}$ are the orbital separation and the mass ratio of the binary, respectively.

The mass transfer rate from the donor star via Roche lobe overflow can be written as

$$
\dot{M}_{2}=-\mathrm{RMT} \cdot \max \left[0,\left(R_{2} / R_{\mathrm{L}}-1\right)^{3}\right] M_{\odot} \mathrm{yr}^{-1},
$$

here we take RMT to be 1000 in the calculations. We neglect the spin angular momentum of LMXBs because it is smaller compared with the total orbital angular momentum of the system ${ }^{1}$. In the calculations we consider two types of mechanisms for orbital angular momentum loss from the binary system, they are described as follows.

\footnotetext{
${ }^{1}$ If we take typical parameters for the binary, such as $M_{1}=$ $1.4 M_{\odot}, M_{2}=1.0 M_{\odot}, R_{1}=10^{6} \mathrm{~cm}, R_{2}=1 R_{\odot}$, the spin period of the NS is $1 \mathrm{~ms}$, and the orbital period is $P_{\text {orb }}=3 \mathrm{~d}$, the orbital angular momentum of the binary is estimated to be $J_{\text {orb }}=1.9 \times 10^{52} \mathrm{~g} \mathrm{~cm}^{2} \mathrm{~s}^{-1}$. Assuming that the donor star corotates with the orbital rotation, we have $J_{1}=7.0 \times 10^{48} \mathrm{~g} \mathrm{~cm}^{2} \mathrm{~s}^{-1}, J_{2}=2 \pi k^{2} M_{2} R_{2}^{2} / P_{\text {orb }}=2.4 \times 10^{49} \mathrm{~g} \mathrm{~cm}^{2} \mathrm{~s}^{-1}$ ( $k^{2} \approx 0.1$ for main-sequence stars, Dervişoğlu et al. 2010), where $J_{1}, J_{2}$ are the spin angular momentum of the NS, and the donor star, respectively. It is clear that the orbital angular momentum is three to four orders of magnitude greater than the spin angular momentum.
}

\subsection{Orbital angular momentum loss mechanisms}

\subsubsection{Magnetic braking}

The low-mass main-sequence donor stars in LMXBs were braked by the coupling between the magnetic field and the stellar winds (Verbunt \& Zwaan 1981). The loss of specific angular momentum in stellar winds is very large because the outflowing ionized particles are bound in the magnetic field lines to corotate with the stars out to the magnetospheric radius (see Weber \& Davis 1967; Mestel \& Spruit 1987; Kalogera 1999). In LMXBs, the spin angular momentum loss of the donor star by magnetic braking causes its spin-down, while the tidal interaction between two components would continuously act to spin the donor star back up into corotation with the orbital motion. Because the spin-up of the donor star consumes the orbital angular momentum, magnetic braking indirectly extracts the orbital angular momentum of LMXBs.

A standard magnetic braking model for the angular momentum loss is widely applied in studying the evolution of cataclysmic variables, and the angular momentum loss rate can be written as (Rappaport et al. 1983)

$$
\dot{J}_{\mathrm{MB}} \simeq-3.8 \times 10^{-30} M_{2} R_{\odot}^{4}\left(R_{2} / R_{\odot}\right)^{\gamma} \omega^{3} \mathrm{dyn} \mathrm{cm},
$$

where $R_{2}$ is the radius, $\omega$ the angular velocity of the donor star, and $\gamma$ is a dimensionless parameter in the range of zero to four.

However, observations based on rapidly rotating stars with a spin period less than two to five days in young open clusters, indicate that the standard magnetic braking description should overestimate the angular momentum loss rate (Queloz 1998; Andronov et al. 2003). Sills et al. (2000) propose the induced magnetic braking model, and the angular momentum loss rate is given by

$$
\dot{J}_{\mathrm{mb}}= \begin{cases}-K \omega^{3}\left(\frac{R_{2}}{R_{\odot}} \frac{M_{\odot}}{M_{2}}\right)^{1 / 2}, & \omega \leq \omega_{\text {crit }} \\ -K \omega \omega_{\text {crit }}^{2}\left(\frac{R_{2}}{R_{\odot}} \frac{M_{\odot}}{M_{2}}\right)^{1 / 2}, & \omega>\omega_{\text {crit }}\end{cases}
$$

where $K=2.7 \times 10^{47} \mathrm{~g} \mathrm{~cm}^{2}$ (Andronov et al. 2003). Observations show that there is a saturation level in the angular momentum loss, and $\omega_{\text {crit }}$ is the critical angular velocity at which the angular momentum loss rate reaches a saturated state. Kim \& Demarque (1996) suggest that $\omega_{\text {crit }}$ is inversely proportional to the convective turbulent timescale in the star at 200 Myr age,

$$
\omega_{\text {crit }}=\omega_{\text {crit } \odot} \frac{\tau_{\odot}}{\tau},
$$

where $\omega_{\text {crit }, \odot}=2.9 \times 10^{-5} \mathrm{~Hz}, \tau_{\odot}$, and $\tau$ are the convective turbulent timescales of the Sun and donor star, respectively (Krishnamurthi et al. 1997).

\subsubsection{Accretion disk instability and mass loss}

According to the mass of PSR J1713+0747 inferred by its Shapiro delay, there is a large amount of transfer material that should be lost from this binary system during the mass exchange. Either the accretion disk instabilities (Pringle 1981; van Paradijs 1996) or the propeller effect (Illarionov \& Sunyaev 1975) may be responsible for the mass loss even for sub-Eddington accretion. During the mass transfer via Roche Lobe overflow, the transferring material would form an accretion disk around the neutron star. It is well known that 
disk accretion in LMXBs may suffer thermal and viscous instability, if the effective temperature in the disk is below the hydrogen ionization temperature about $6500 \mathrm{~K}$ (van Paradijs 1996; King et al. 1997; Lasota 2001). This corresponds to a critical mass-transfer rate under which the accretion disk is unstable. This critical mass-transfer rate is (van Paradijs 1996; Dubus et al. 1999)

$$
\dot{M}_{\mathrm{cr}} \simeq 3.2 \times 10^{-9}\left(\frac{M_{1}}{1.4 M_{\odot}}\right)^{0.5}\left(\frac{M_{2}}{1 M_{\odot}}\right)^{-0.2}\left(\frac{P_{\text {orb }}}{1 \mathrm{~d}}\right)^{1.4} M_{\odot} \mathrm{yr}^{-1}
$$

When an accretion-disk instability occurs, the accreting neutron star will become a transient X-ray source, experiencing short-lived outbursts separated by long-term quiescence. When the mass transfer rate $-\dot{M}_{2}$ is lower than $\dot{M}_{\mathrm{cr}}$, we assume that the neutron star accretes only during outbursts, so the accretion rate is $\dot{M}_{\text {ac }} t_{\text {out }}=-\dot{M}_{2} t_{\mathrm{r}}$, where $t_{\text {out }}, t_{\mathrm{r}}$ are the outburst timescale and the recurrence time, respectively. Introducing the duty cycle $f=t_{\text {out }} / t_{\mathrm{r}}$, we have $\dot{M}_{\mathrm{ac}}=-\dot{M}_{2} / f$ [a typical duty cycle is in the range of $0.1-0.001$ (King et al. 2003)]. If $-\dot{M}_{2} \geq \dot{M}_{\mathrm{cr}}$, the accretion rate is $\dot{M}_{\text {ac }}=-\dot{M}_{2}$. In addition, in both cases the mass growth rate of the neutron star $\dot{M}_{1}$ is limited to the Eddington accretion rate $\dot{M}_{\text {Edd }}$, i.e.

$$
\dot{M}_{1}=\min \left[\dot{M}_{\mathrm{ac}}, \dot{M}_{\mathrm{Edd}}\right] \text {, }
$$

where $\dot{M}_{\text {Edd }} \approx 1.5 \times 10^{-8} M_{\odot} \mathrm{yr}^{-1}$ is the Eddington accretion rate of the neutron star.

The transferred matter that exceeds the Eddington accretion rate is assumed to be ejected in the vicinity of the neutron star. The matter forms an isotropic wind and carries away the specific orbital angular momentum of the neutron star. Based on the above assumptions, we can derive the angular momentum loss rate:

$$
\dot{J}_{\mathrm{IW}}=\frac{\dot{M} M_{2}}{M M_{1}} J
$$

where $J=a^{2} \mu \Omega$ is the total orbital angular momentum of the LMXB with a circular orbit, $\mu=M_{1} M_{2} /\left(M_{1}+M_{2}\right), M=M_{1}+$ $M_{2}$, and $\dot{M}=\dot{M}_{1}-\dot{M}_{\mathrm{ac}}$ are the reduced mass, the total mass, and the mass-loss rate of the binary system, respectively.

\subsection{WD regime: the evolutionary code}

When the LMXB becomes a detached system, the donor star evolves into an He-core WD. From this stage, during thermonuclear flashes scenario and in WD regime, we adopt the stellar evolutive code LPCODE (Althaus et al. 2005). The code is based on a detailed description of the main physical processes involved in the formation of WD. Radiative opacities are those from OPAL (including carbon- and oxygen-rich compositions) for arbitrary metallicity (Iglesias \& Rogers 1996). These opacities are calculated for metallicities consistent with the diffusion predictions. During the WD cooling regime gravitational settling leads to metal-depleted outer layers. The equation of state is an updated version of that of Magni \& Mazzitelli (1979). High-density conductive opacities and the various mechanisms of neutrino emission are taken from works of Itoh et al. (1992). Hydrogen burning is taken into account by considering a complete network of thermonuclear reaction rates corresponding to the proton-proton chain and $\mathrm{CNO}$ cycle. Nuclear reactions rates are taken from Caughlan \& Fowler (1988). We consider the evolution of the chemical abundance distribution caused by element diffusion during the WD regime. Our treatment of

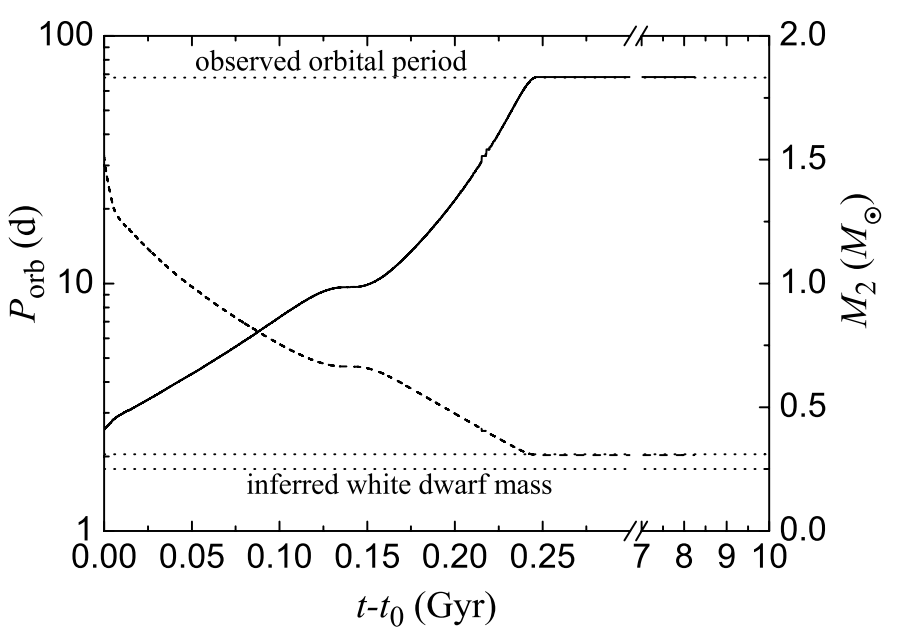

Fig. 1. Evolution of the orbital period (solid curve) and the donor star mass (dashed curve) vs. evolutive timescale for LMXB. LMXB has a donor star with an initial mass of $1.5 M_{\odot}$ and an initial orbital period of $2.57 \mathrm{~d}$. Horizontal dotted lines on the bottom of the figure correspond to the observed mass of WD with its error, and horizontal dotted line on the top of the figure represents the observed orbital period.

time-dependent diffusion is based on the multicomponent gas treatment presented by Burgers (1969), for element diffusion scheme we considered the following nuclear species ${ }^{1} \mathrm{H},{ }^{3} \mathrm{He}$, ${ }^{4} \mathrm{He},{ }^{12} \mathrm{C},{ }^{14} \mathrm{~N}$, and ${ }^{16} \mathrm{O}$. Abundance changes are computed according to element diffusion and then to nuclear reactions and convective mixing.

In this work, we have computed the complete evolution of seven remnants arisen from binary systems as soon as the transfer of mass has ceased. The masses of these seven pre-WDs are listed in Table $1\left(M_{2, \mathrm{f}}\right)$. The evolution of these WDs is continued until they arrive at a luminosity of $\log \left(L / L_{\odot}\right) \approx-4.8$.

\section{Results}

To study the characteristics of the progenitor of PSR J1713+0747, we calculated evolutionary sequences of many LMXBs. We consider both the angular momentum loss mechanisms described in Sect. 2.1, adopting the induced magnetic braking model (Eq. (4)) for the donor stars with mass less than $1.5 M_{\odot}$. Some initial input parameters were set as follows, the neutron star has a canonical mass of $M_{1, \mathrm{i}}=1.4 M_{\odot}$, the mass of the donor star $M_{2, \mathrm{i}}$ is in the range of 1.0-2.0 $M_{\odot}$ (with a solar metallicity of $Z=0.02$ ), the duty cycle $f=0.01$.

The characteristic age of PSR J1713+0747 estimated by Splaver et al. (2005) is $\tau_{\mathrm{PSR}} \approx 8$ Gyr. This implies that the WD has a cooling timescale $\tau_{\text {cool }}$ of approximately $8 \mathrm{Gyr}$ since the end of the mass transfer episode. Based on different initial orbital period $P_{\mathrm{orb}, i}$, we stop the binary evolution calculation when the binary becomes a detached system and the donor star evolve into a WD. Subsequently, a WD evolutionary code (LPCODE) is applied to the donor star. If the final donor star mass is in the range of 0.25 to $0.31 M_{\odot}$, and the orbital period of the binary is approximately of $67.8 \mathrm{~d}$, the corresponding binary was assumed to be the progenitor candidate of PSR J1713+0747.

Firstly, we calculated the evolutionary sequences of an LMXB with $M_{2, \mathrm{i}}=1.5 M_{\odot}$ and $P_{\text {orb,i }}=2.57$ d. In Figs. 1 and 2 we plot the evolution of orbital period and donor star mass, and the mass transfer rate and the neutron star mass, respectively. In the early phase, the orbital period decrease to be 


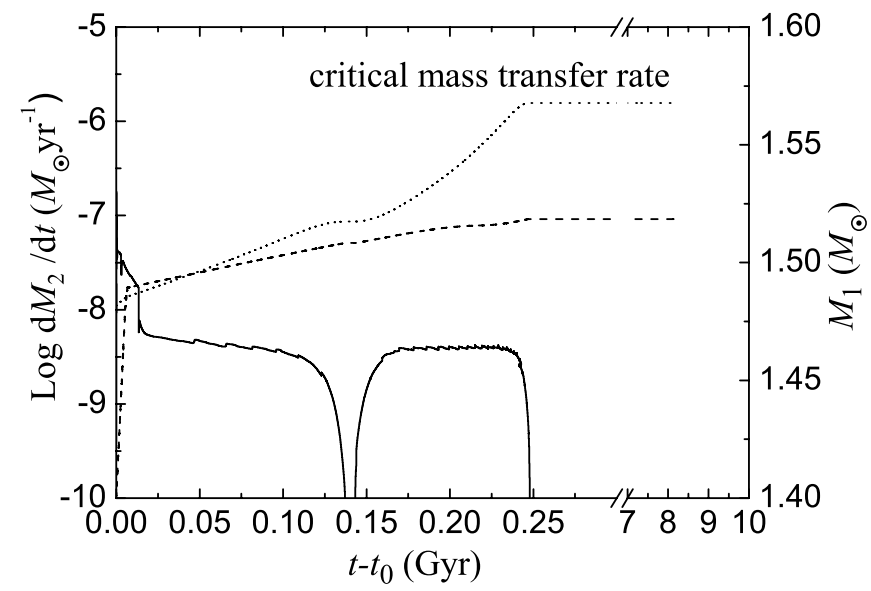

Fig. 2. Evolution of the mass transfer rate (solid curve) and the neutron star mass (dashed curve) with the evolutive timescale for the same LMXB of Fig. 1. The dotted curve represents the critical mass transfer rate, under which the disk accretion is unstable. The mass transfer rate has been slightly smoothed for clarity.

approximately $2.56 \mathrm{~d}$ due to magnetic braking even if there is no mass exchange. With nuclear evolution, the donor star fills its Roche lobe when its age is $2.54 \mathrm{Gyr}$. When $M_{2}=0.66 M_{\odot}$, and $P_{\text {orb }}=9.66 \mathrm{~d}$, the binary system detaches and mass transfer ceases about 4.9 Myr. This detached phase also is found in other studies for the evolution of LMXBs (Tauris \& Savonije 1999; Han et al. (2000); Podsiadlowski et al. 2002). After the temporary shrinkage, the donor star fills its Roche lobe again and climbs the giant branch. When the age is $2.79 \mathrm{Gyr}$, the second Roche lobe overflow (RLOF) episode terminates. Hereafter, the donor star evolves to a helium WD and cools. The evolution end point is a binary pulsar with an orbital period of $68.3 \mathrm{~d}$. The binary consists of an He-core WD of $0.3070 M_{\odot}$ and a neutron star of $1.5184 M_{\odot}$. Because the material is transferred from the more massive donor star to the less massive neutron star, the mass transfer proceeds initially on short thermal timescale about 10 Myr. In this phase, the mass transfer rate is in a range of $10^{-7}$ to $10^{-8} M_{\odot} \mathrm{yr}^{-1}$, and the LMXB is a short-lived persistent X-ray source. Subsequently, the mass transfer occurs on a much longer nuclear timescale of $0.25 \mathrm{Gyr}$, with a rate of $10^{-9}$ or $10^{-8} M_{\odot} \mathrm{yr}^{-1}$. This rate is always less than the critical mass transfer rate. Therefore, the progenitor of PSR J1713+0747 should be a transient X-ray source about $95 \%$ of all its life.

In Fig. 3, we plot the evolutionary path of the donor star in the Hertzsprung-Russell diagram (HRD). After the donor star evolves into a pre-WD, a hydrogen thermonuclear flash occurs. During this stage the element diffusion is a key physical ingredient in obtaining a thin hydrogen envelope. We stress that thermonuclear flashes also occur in the absence of element diffusion, but in this case the residual hydrogen envelope is thick, according to Benvenuto \& De Vito (2004) and Panei et al. (2007). Figure 4 presents theoretical predicted relation between $P_{\text {orb,f }}$ and $M_{2, \mathrm{f}}$ for binary radio pulsars. It is clear that our simulation result is consistent with the theoretical prediction.

To investigate the distribution of the initial donor star mass and orbital period for the progenitor system of PSR J1713+0747, we calculated the evolution of a large amount of LMXBs for different initial input parameters. Table 1 summarizes the calculated results. Assuming that the cooling timescale of the WD is $\tau_{\text {cool }}=8 \mathrm{Gyr}$, as seen in Table 1 makes it clear that the donor star with an initial mass of $M_{2, \mathrm{i}}=1.0-1.2 M_{\odot}$ would require

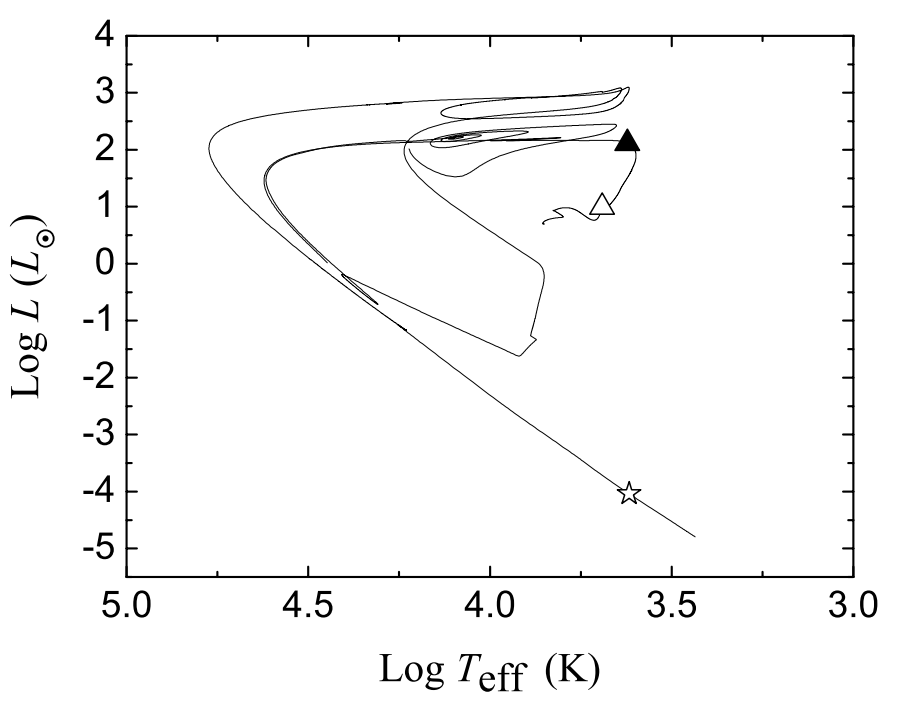

Fig. 3. Calculated evolutionary tracks in $\mathrm{H}-\mathrm{R}$ diagram for the donor star with an initial mass of $1.5 M_{\odot}$. The open triangle, solid triangle, and open star indicate the beginning of RLOF, the end of the last episode of RLOF and the present situation of the donor star, respectively.

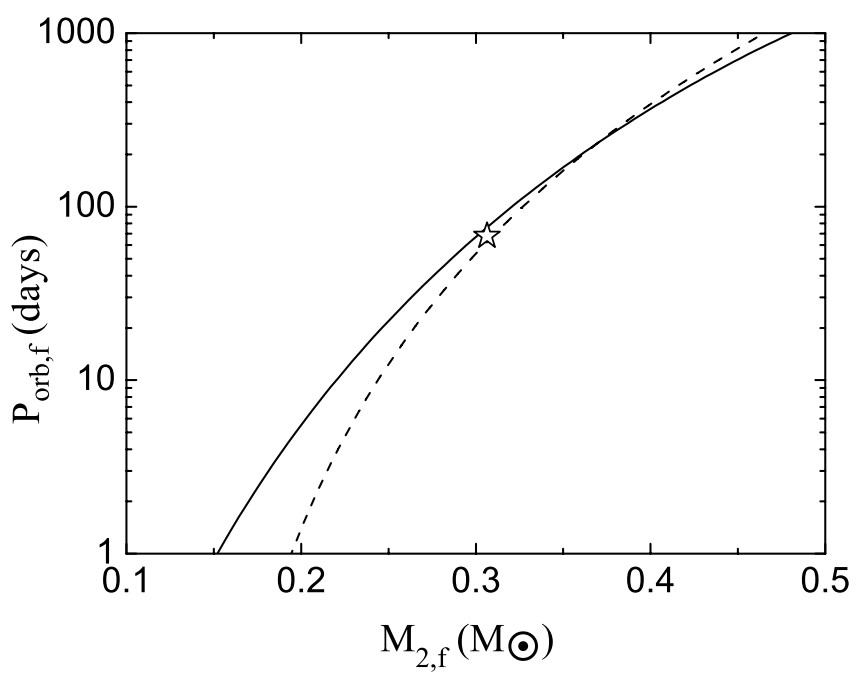

Fig. 4. Theoretical predicted relation between $P_{\text {orb,f }}$ and $M_{2, \mathrm{f}}$ for binary radio pulsars. The solid curves and dashed curves correspond to the relations obtained by Rappaport et al. (1995) and Tauris \& Savonije (1999). The open star sign represents our simulation result.

times in excess of the Hubble time in order to evolve into a dim WD like the one observed in PSR J1713+0747. Therefore, PSR $\mathrm{J} 1713+0747$ may originated from an LMXB with a donor star of $M_{2, \mathrm{i}}=1.3-1.6 M_{\odot}$ and an initial orbital period of $P_{\text {orb,i }}=$ $2.40-4.15 \mathrm{~d}$. Thus with an age of $\tau_{\text {cool }}=8 \mathrm{Gyr}$, we obtain WD effective temperatures in the range of $\sim 3870-4120 \mathrm{~K}$, approximately in agreement with the observational datum $3700 \pm$ $100 \mathrm{~K}$ given by Lundgren et al. (1996), Benvenuto et al. (2006) derived a value of $4320 \pm 180 \mathrm{~K}$ and $4250 \pm 250 \mathrm{~K}$ determined by Schönberner et al. (2000). In addition, the luminosity of the WD was determined to be $-4.171 \leq \log \left(L / L_{\odot}\right) \leq-4.047$. Apparently, the final effective temperature of the WD in our calculations is sensitive to its cooling timescale, and a long cooling timescale can result in a low effective temperature. On the basis of these models with real history, we estimated the range 
Table 1. Calculated results for different initial input parameters.

\begin{tabular}{lllllllllllll}
\hline \hline No. & $\begin{array}{l}M_{2, \mathrm{i}} \\
\left(M_{\odot}\right)\end{array}$ & $\begin{array}{l}P_{\text {orb, } i} \\
(\mathrm{days})\end{array}$ & $\begin{array}{l}t_{0} \\
(\mathrm{Gyr})\end{array}$ & $\begin{array}{l}t_{\mathrm{f}} \\
(\mathrm{Gyr})\end{array}$ & $\begin{array}{l}t_{\mathrm{f}}-t_{0} \\
(\mathrm{Gyr})\end{array}$ & $\begin{array}{l}M_{2, \mathrm{f}} \\
\left(M_{\odot}\right)\end{array}$ & $\begin{array}{l}M_{1, \mathrm{f}} \\
\left(M_{\odot}\right)\end{array}$ & $\begin{array}{l}P_{\text {orb }, f} \\
(\mathrm{days})\end{array}$ & $\begin{array}{l}t_{\mathrm{p}, 8} \\
(\mathrm{Gyr})\end{array}$ & $\begin{array}{l}\log L_{8} \\
\left(L_{\odot}\right)\end{array}$ & $\begin{array}{l}T_{\text {eff, }, 8} \\
(\mathrm{~K})\end{array}$ & $\begin{array}{l}\log g_{8} \\
\left(\mathrm{~cm} \mathrm{~s}^{-2}\right)\end{array}$ \\
\hline 1 & 1.0 & 5.65 & 12.48 & 12.68 & 0.20 & 0.3058 & 1.4211 & 66.7 & 20.68 & -4.0318 & 4152 & 7.3810 \\
2 & 1.1 & 5.09 & 8.74 & 8.95 & 0.21 & 0.3069 & 1.4220 & 68.8 & 16.95 & -4.1054 & 3999 & 7.3909 \\
3 & 1.2 & 4.48 & 6.12 & 6.34 & 0.22 & 0.3063 & 1.4247 & 67.6 & 14.34 & -4.1349 & 3937 & 7.3925 \\
4 & 1.3 & 4.15 & 4.70 & 4.92 & 0.22 & 0.3067 & 1.4260 & 68.1 & 12.92 & -4.1114 & 3985 & 7.3905 \\
5 & 1.4 & 3.70 & 3.30 & 3.54 & 0.24 & 0.3068 & 1.4964 & 68.0 & 11.54 & -4.1707 & 3872 & 7.4001 \\
6 & 1.5 & 2.57 & 2.54 & 2.79 & 0.25 & 0.3070 & 1.5184 & 68.3 & 10.79 & -4.0471 & 4122 & 7.3856 \\
7 & 1.6 & 2.40 & 1.96 & 2.20 & 0.24 & 0.3068 & 1.5383 & 67.5 & 10.20 & -4.1682 & 3880 & 7.4010 \\
\hline
\end{tabular}

Notes. The meaning of the columns are presented as follows. $M_{2, \mathrm{i}}$ : initial mass of the donor star; $P_{\mathrm{orb}, i}:$ initial orbital period; $t_{0}$ : age when Roche lobe overflow begins; $t_{\mathrm{f}}$ : age when the main Roche lobe overflow ends; $t_{\mathrm{f}}-t_{0}$ : mass transfer timescale; $M_{2, \mathrm{f}}:$ mass of the WD; $M_{1, \mathrm{f}}$ : mass of the neutron star; $P_{\mathrm{orb}, \mathrm{f}}$ : final orbital period; $t_{\mathrm{p}, 8}, \log L_{8}, T_{\mathrm{eff}, 8}$ and $\log g_{8}:$ age, luminosity, effective temperature and gravity when $\tau_{\mathrm{co}}=8 \mathrm{Gyr}$, respectively.

of surface gravity for the WD in $7.386 \leq \log (g) \leq 7.401(\mathrm{~g}$ in units of $\mathrm{g} \mathrm{cm}^{-2}$ ). Detailed tabulations of our WD evolutionary sequences are available at http://www. fcaglp.unlp.edu.ar/ $\sim$ panei/papers.html.

Although the final mass of the neutron star (see Nos. 6 and 7 in Table 1) is slightly higher than the one derived by Splaver et al. 2005, it is still in the allowed mass interval by theoretical orbital period - WD mass relation $\left(M_{1}=\right.$ $\left.1.53_{-0.06}^{+0.08} M_{\odot}\right)$. Though adopting various scenarios, our results are approximately consistent with the ones derived by Benvenuto et al. (2006).

\section{Summary and discussion}

In this paper, we attempt to carefully study the evolution history and the progenitor characteristics of PSR J1713+0747. Based on Eggleton's stellar evolution code, we calculated the evolutionary sequences of a number of LMXBs to fit the observed data $\left(M_{1}, M_{2}\right.$, and $\left.P_{\text {orb }}\right)$ of PSR J1713+0747. For the WD regime we employed the stellar evolution code LPCODE (Althaus et al. 2005).

In calculations, angular momentum loss via magnetic braking is included if the mass of the donor star is less than $1.5 \mathrm{M}_{\odot}$. The observations show that PSR J1713+0747 has only accreted less than $0.1 M_{\odot}$ from the donor star during evolution. Unstable mass transfer due to the thermal and viscous instability of the accretion disk may be responsible for the extensive mass $\operatorname{loss}^{2}$. Therefore, LMXBs may be transient X-ray sources for most of their life. If we adopt a duty cycle of 0.01 , the progenitor of PSR J1713+0747 can be constrained to be an LMXB with an initial donor star of 1.3-1.6 $M_{\odot}(Z=0.02)$ and an initial orbital period of 2.40-4.15 d. Adopting a cooling timescale of 8 Gyr for the WD, our calculations show that at present its effective temperature is between 3870 and $4120 \mathrm{~K}$. Compared with the results derived by Benvenuto et al. (2006), for $Z=0.02$, the cooling timescale of the WD in our model is close to the spindown age of the pulsar ${ }^{3}$. We obtained absolute magnitudes of $M_{V} \approx 15.7 \pm 0.4$ and $M_{I} \approx 13.8 \pm 0.3$ in the $V$ and $I$ bands, and inferred effective temperatures $T_{\text {eff }} \approx 3301-4169 \mathrm{~K}$ and $T_{\text {eff }} \approx 3946-4694 \mathrm{~K}$. Detailed tabulations of our WD evolutionary sequences are available at http://www . fcaglp. unlp. edu. ar/ panei/papers.html.

\footnotetext{
${ }^{2}$ Certainly, the propeller effect of the pulsar cannot be excluded (Illarionov \& Sunyaev 1975).

${ }^{3}$ Benvenuto et al. (2006) propose that the WD needs $10.8 \pm 2$ Gyr to cool when $Z=0.02$.
}

Our accretion disk instability model successfully reproduces the orbital period and the mass of the WD. However, the effective temperature of the WD is slightly higher than observations, although we consider gravitational settling and chemical and thermal diffusion during the evolution of the WD (Panei et al. 2007). Employing Hubble Space Telescope (HST), Lundgren et al. (1996) performed optical observations for the WD companion of PSR J1713+0747. Adopting the temperature calibration of $(V-I)$ colour given by Monet et al. (1992), they presented the effective temperature of the WD is $T_{\text {eff }}=3700 \pm 100 \mathrm{~K}$. Assuming a blackbody spectrum, Hansen \& Phinney (1998) fitted the HST data, and obtained $T_{\text {eff }}=3430 \pm 270 \mathrm{~K}$. A long cooling timescale of the WD may be responsible for the discrepancy between our evolution results and the inferred value of observations. In this work we take $\tau_{\text {co }}=8$ Gyr (Splaver et al. 2005). However, it is very rough to estimate the spin-down age of the pulsar from $\tau_{\mathrm{PSR}}=P / 2 \dot{P}$, where $P$ and $\dot{P}$ are the spin period and the period derivative of the pulsar, respectively ${ }^{4}$. We expect that further spectroscopic and photometric observations can help us understand the evolutionary track of PSR J1713+0747.

As an alternative evolution channel, millisecond pulsars may also be formed by accretion-induced collapse (AIC) when the mass of the accreting ONe WD exceeds the Chandrasekhar mass of $1.4 M_{\odot}$ (Nomoto 1984, Nomoto 1986). Statistical analysis shows that the birthrate of LMXBs is 1-2 orders of magnitude lower than that of millisecond pulsars (Cote \& Pylyser 1989, Lorimer 1995). The AIC channel was raised to interpret this birthrate discrepancy (Bailyn \& Grindlay 1990). Based on a scenario including both the AIC of an accreting WD and unstable disk accretion, Xu \& Li (2009) propose that GRO J1744-28 may be evolved from a WD binary. Recently, the estimated birthrates of millisecond pulsars show that the often neglected AIC channel cannot be ignored (Hurley et al. 2010). Therefore, the formation possibility of PSR J1713+0747 via the AIC of a massive WD cannot be ruled out. We plan to explore the AIC channel for the formation and evolution of PSR J1713+0747.

Of course, there are many uncertainties in our input physics. Firstly, the orbital angular momentum loss plays a vital role in the evolution of LMXBs. Some mechanism of extracting angular momentum possibly exists besides magnetic braking and

\footnotetext{
${ }^{4}$ The standard spin-down age of the pulsar is $\tau_{\mathrm{PSR}}=\frac{P}{(n-1) P}[1-$ $\left(P_{0} / P\right)^{n-1}$, where $P_{0}$ is the initial period and $n$ the braking index of the pulsar. When $P_{0} \ll P$ and $n=3, \tau_{\mathrm{PSR}}=P / 2 \dot{P}$. However, the derived braking indice of several young radio pulsars are all less than 3 (Xu \& Qiao 2001; Chen \& Li 2006).
} 
mass loss due to the accretion disk instability. For example, the propeller effect of the neutron star may carry away the specific orbital angular momentum at the magnetosphere of the neutron star (Illarionov \& Sunyaev 1975). In addition, a circumbinary disk around LMXBs may also extract orbital angular momentum efficiently from the binary system (Spruit \& Taam 2001; Taam \& Spruit 2001). Secondly, our results strongly depend on duty cycles, which are poorly known. In this work, we take a constant duty cycle. However, duty cycles may change from one system to another (Lasota 2001), and change with the orbital period and the mass transfer rate. Finally, a gamma-ray flux caused by the interaction of the magnetic field of the neutron star with the accretion disk may drive a wind from the donor star by heating its outer layers; i.e., the donor star may be evaporated (van den Heuvel \& van Paradijs 1988; Kluźiniak et al. 1988; Ruderman et al. 1989a; Ruderman et al. 1989b).

Acknowledgements. We are grateful to the referee, Christopher A. Tout, for his constructive suggestions for improving this manuscript. We also thank ZhanWen Han and Xue-Fei Chen for their helpful discussions, and thank Stephen Justham for his help in improving the English of this manuscript. This work has been supported in part by the National Natural Science Foundation of China (Grant No 10873011), Program for Science \& Technology Innovation Talents in Universities of Henan Province, and Innovation Scientists and Technicians Troop Construction Projects of Henan Province, China. We acknowledge the Facultad de Ciencias Astronómicas y Geofísicas (UNLP), Instituto de Astrofísica de La Plata (CCT La Plata, CONICET-UNLP) and Lic. H. Viturro for his technical support.

\section{References}

Alexander, D. R., \& Ferguson, J. W. 1994, ApJ, 437, 879

Alpar, M. A., Cheng, A. F., Ruderman, M. A., \& Shaham, J. 1982, Nature, 300, 728

Althaus, L. G., Serenelli, A. M., Panei, J. A., et al. 2005, A\&A, 435, 631

Andronov, N., Pinsonneault, M., \& Sills, A. 2003, ApJ, 582, 358

Bailyn C. D., \& Grindlay J. E. 1990, ApJ, 353, 159

Benvenuto, O. G., \& De Vito, M. A. 2004, MNRAS, 352, 249

Benvenuto, O. G., Rohrmann, R. D., \& De Vito, M. A. 2006, MNRAS, 366, 1520

Bhattacharya, D., \& van den Heuvel, E. P. J. 1991, Phys. Rep., 203, 1

Burgers, J. M. 1969, Flow Equations for Composite Gases (New York: Academic Press)

Camilo, F., Foster, R. S., \& Wolszczan, A. 1994, ApJ, 437, L39

Caughlan, G. R., \& Fowler, W. A. 1988, Atomic Data and Nuclear Data Tables, 40,284

Chen, W.-C., \& Li, X.-D. 2006, A\&A, 450, L1

Chen, W.-C., Li, X.-D., \& Wang, Z.-R. 2006, PASJ, 58, 153

Cote J., \& Pylyser, E. H. P. 1989, A\&A, 218, 131

Dervişoğlu, A., Tout, C. A., \& Ibanoğlu, C. 2010, MNRAS, 406, 1071

Dubus, G., Lasota, J.-P., Hameury, J.-M., \& Charles, P. 1999, MNRAS, 303, 139

Eggleton, P. P. 1971, MNRAS, 151, 351

Eggleton, P. P. 1972, MNRAS, 156, 361

Eggleton, P. P. 1983, ApJ, 268, 368
Foster, R. S., Wolszczan, A., \& Camilo F. 1993, ApJ, 410, L91

Giacconi, R., Gursky, H., Paolini, F. R., \& Rossi, B. B. 1962, Phys. Rev. Lett., 9, 439

Han, Z., Podsiadlowski, Ph., \& Eggleton, P. P. 1994, MNRAS, 270, 121

Han, Z., Tout, C. A., \& Eggleton, P. P. 2000, MNRAS, 319, 215

Hansen, B. M. S., \& Phinney, E. S. 1998, MNRAS, 294, 569

Hurley J. R., Tout C. A., Wickramasinghe, D. T., Ferrario, L., \& Kiel, P. D. 2010, MNRAS, 402, 1437

Iglesias, C. A., \& Rogers, F. J. 1996, ApJ, 464, 943

Illarionov, A. F., \& Sunyaev, R. A. 1975, A\&A, 39, 185

Itoh, N., Mutoh, H., Hikita, A., \& Kohyama, Y. 1992, ApJ, 395, 622; Erratum: 1993, ApJ, 404, 418

Kalogera, V. 1999, ApJ, 521, 723

Kim, Y.-C., \& Demarque, P. 1996, ApJ, 457, 340

King, A. R., Frank, J., Kolb, U., \& Titter, H. 1997, ApJ, 484, 844

King, A. R., Rolfe, D. J., \& Schenker, K. 2003, MNRAS, 341, L35

Kluźiniak, W., Ruderman, M., Shaham, J., \& Tavani, M. 1988, Nature, 334, 225

Krishnamurthi, A., Pinsonneault, M. H., Barnes, S., \& Sofia, S. 1997, ApJ, 480, 303

Lasota, J.-P. 2001, NewAR, 45, 449

Lundgren, S. C., Foster, R. S., \& Camilo, F. 1996, in Pulsars: Problems and Progress (San Francisco), ASP Conf. Ser. 105, IAU Colloq., 160, 497

Lorimer, D. R. 1995, MNRAS, 274, 300

Magni, G., \& Mazzitelli, I. 1979, A\&A, 72, 134

Mestel, L., \& Spruit, H. C. 1987, MNRAS, 226, 57

Monet, D. G., Dahn, C. C., Vrba, F. J., et al. 1992, AJ, 103, 638

Nomoto, K. 1984, in Problems of Colalpse and Numerical Relativity, ed. D. Bancel, \& M. Sifnore (Dordrecht: Reidel), 89

Nomoto K. 1986, in Accretion Processes in Astrophysics, ed. J. Audouze, J. Tran Thanh Van (Gif-sur-Yvette: Éditions Frontières), 137

Novikov, I. D., \& Zel'Dovitch, Y. B. 1966, Nuova Cimento Sup., 4, 810

Panei, J. A., Althaus, L. G., Chen, X., \& Han, Z. 2007, MNRAS, 382, 779

Pfahl, E., Rappaport, S., \& Podsiadlowski, Ph. 2002, ApJ, 573, 283

Podsiadlowski, Ph., Rappaport, S., \& Pfahl, E. D. 2002, ApJ, 565, 1107

Pols, O., Tout, C. A., Eggleton, P. P., \& Han, Z. 1995, MNRAS, 274, 964

Pringle, J. E. 1981, ARA\&A, 19, 137

Queloz, D., Allain, S., Mermilliod, J. C., Bouvier, J., \& Mayor, M. 1998, A\&A, 335,183

Rappaport, S., Verbunt, F., \& Joss, P. C. 1983, ApJ, 275, 713

Rappaport, S., Podsiadlowski, Ph., Joss, P. C., Di Stefano, R., \& Han, Z. 1995, MNRAS, 273, 731

Ruderman, M., Shaham, J., \& Tavani, M. 1989a, ApJ, 336, 507

Ruderman, M., Shaham, J., Tavani, M., \& Fichler, D. 1989b, ApJ, 343, 292

Rogers, F. J., \& Iglesias, C. A. 1992, ApJS, 79, 507

Schönberner, D., Driebe, T., \& Blöcker, T. 2000, A\&A, 356, 929

Shklovskii, I. 1967, ApJ, 148, L1

Sills, A., Pinsonneault, M. H., \& Terndrup, D. M. 2000, ApJ, 534, 335

Splaver, E. M., Nice, D. J., Stairs, I. H., et al. 2005, ApJ, 620, 405

Spruit, H. C., \& Taam, R. E. 2001, ApJ, 548, 900

Stairs, I. H. 2004, Science, 304, 547

Taam, R. E., \& Spruit, H. C. 2001, ApJ, 561, 329

Tauris, T. M., \& Savonije, G. J. 1999, A\&A, 350, 928

van den Heuvel, E. P. J., \& van Paradijs, J. 1988, Nature, 334, 227

van Paradijs, J. 1996, ApJ, 464, L139

Verbunt, F., \& Zwaan, C. 1981, A\&A, 100, L7

Weber, E. J., \& Davis, L. 1967, ApJ, 148, 217

Wijnards, R., \& van der Klis, M. 1998, Nature, 394, 344

Xu, R. X., \& Qiao, G. J. 2001, ApJ, 561, L85

Xu, X. -J., \& Li, X. -D. 2009, A\&A, 495, 243

Zeldovich, Ya. B., \& Guseynov, O. H. 1966, ApJ, 144, 840 